\title{
AMP-forming acetyl-CoA synthetases in Archaea show unexpected diversity in substrate utilization
}

\author{
CHERYL INGRAM-SMITH ${ }^{1}$ and KERRY S. SMITH ${ }^{1,2}$ \\ ${ }^{1}$ Department of Genetics and Biochemistry, Clemson University, Clemson, SC 29634-0318, USA \\ ${ }^{2}$ Corresponding author (kssmith@clemson.edu)
}

Received May 15, 2006; accepted August 14, 2006; published online...

\begin{abstract}
Summary Adenosine monophosphate (AMP)-forming acetyl-CoA synthetase (ACS; acetate:CoA ligase (AMP-forming), EC 6.2.1.1) is a key enzyme for conversion of acetate to acetyl-CoA, an essential intermediate at the junction of anabolic and catabolic pathways. Phylogenetic analysis of putative short and medium chain acyl-CoA synthetase sequences indicates that the ACSs form a distinct clade from other acyl-CoA synthetases. Within this clade, the archaeal ACSs are not monophyletic and fall into three groups composed of both bacterial and archaeal sequences. Kinetic analysis of two archaeal enzymes, an ACS from Methanothermobacter thermautotrophicus (designated as MT-ACS1) and an ACS from Archaeoglobus fulgidus (designated as AF-ACS2), revealed that these enzymes have very different properties. MT-ACS1 has nearly 11 -fold higher affinity and 14-fold higher catalytic efficiency with acetate than with propionate, a property shared by most ACSs. However, AF-ACS2 has only 2.3 -fold higher affinity and catalytic efficiency with acetate than with propionate. This enzyme has an affinity for propionate that is almost identical to that of MT-ACS1 for acetate and nearly tenfold higher than the affinity of MT-ACS1 for propionate. Furthermore, MT-ACS1 is limited to acetate and propionate as acyl substrates, whereas AF-ACS2 can also utilize longer straight and branched chain acyl substrates. Phylogenetic analysis, sequence alignment and structural modeling suggest a molecular basis for the altered substrate preference and expanded substrate range of AF-ACS2 versus MT-ACS1.
\end{abstract}

Keywords: acetate, Archaeoglobus fulgidus, Methanothermobacter thermautotrophicus.

\section{Introduction}

Acetyl-CoA plays a central role in carbon metabolism in the Bacteria, Archaea and Eukarya as an essential intermediate at the junction of various anabolic and catabolic pathways. Adenosine monophosphate (AMP)-forming acetyl-CoA synthetase (ACS; acetate:CoA ligase (AMP-forming), EC 6.2.1.1) is widespread in all three domains of life and is the predominant enzyme for activation of acetate to acetyl-CoA (Equation 1). acetate $+\mathrm{ATP}+\mathrm{CoA} \leftrightarrow$ acetyl-CoA $+\mathrm{AMP}+\mathrm{PP}_{\mathrm{i}}$

Based on isotopic exchange, labeling experiments and detection of an enzyme-bound acetyl-AMP, a mechanism (Equations $2 \mathrm{a}$ and $2 \mathrm{~b}$ ) in which the reaction proceeds through an acetyl-AMP intermediate has been proposed (Berg 1956a, Berg 1956b, Webster 1963, Anke and Spector 1975):

$$
\mathrm{E}+\text { acetate }+\mathrm{ATP} \leftrightarrow \text { Eacetyl-AMP }+\mathrm{PP}_{\mathrm{i}}
$$

Eacetyl-AMP + HSCoA $\leftrightarrow$ E + acetyl-CoA + AMP (2b)

The first step of the reaction, which requires acetate and ATP, but not CoA, involves formation of the acetyl-AMP intermediate and release of pyrophosphate $\left(\mathrm{PP}_{\mathrm{i}}\right)$. In the second step, the acetyl group is transferred to the sulfhydryl group of CoA and AMP is released. An inorganic pyrophosphatase draws the reaction in this forward direction by removing $\mathrm{PP}_{\mathrm{i}}$, a potent inhibitor of ACS.

The ACS is a member of the acyl-adenylate forming enzyme superfamily in which all members undergo a similar two-step reaction mechanism with an enzyme-bound acyl-adenylate intermediate formed in the first step of the reaction. Although members of this superfamily all catalyze mechanistically similar reactions, they share little identity and similarity in amino acid sequence with the exception of a few signature motifs and conserved core sequence motifs (Babbitt et al. 1992, Kleinkauf and Von Dohren 1996, Chang et al. 1997, Marahiel et al. 1997). Structures for several members of this family have been determined (Conti et al. 1996, Conti et al. 1997, May et al. 2002), but provide little information regarding the active site and catalytic mechanism of ACS, because they catalyze unrelated reactions in which the intermediates serve different functions and share too little homology to allow structural modeling of ACS.

The structures of the Salmonella enterica ACS and Saccharomyces cerevisiae ACS1 now provide direct insight into the catalytic mechanism of ACS. The $S$. cerevisiae enzyme was crystallized in the presence of ATP (Jogl and Tong 2004) and the $S$. enterica enzyme (Gulick et al. 2003) was crystallized in the presence of $\mathrm{CoA}$ and adenosine-5'-propyl- 
phosphate, which mimics the acyl-adenylate intermediate (Grayson and Westkaemper 1988, Horswill and EscalanteSemerena 2002). These structures demonstrate the enzyme in two different conformations. The structure of the yeast enzyme is thought to represent the conformation of the enzyme in the first step of the reaction, which involves acetate and ATP but not CoA. In this structure, the smaller $\mathrm{C}$-terminal domain is in an open position away from the active site. The structure of the bacterial enzyme is thought to represent the conformation for the second step of the reaction, in which the acetyladenylate intermediate reacts with CoA. In this structure, the C-terminal domain has rotated $140^{\circ}$ toward the N-terminal domain, thus rearranging the active site upon CoA binding for catalysis of the second step of the reaction.

Most ACSs have a limited substrate range, showing a strong preference for acetate as the acyl substrate, although propionate can serve as a less efficient substrate. However, the Pyrobaculum aerophilum ACS (PA-ACS) has been shown to utilize butyrate and isobutyrate in addition to acetate and propionate (Brasen et al. 2005). Furthermore, PA-ACS is octameric, unlike other ACSs which have been shown to be monomeric, dimeric or trimeric (Brasen et al. 2005). These findings call into question whether ACSs are more diverse than previously expected.

We report here the biochemical and kinetic characterization of two ACSs from the archaea Methanothermobacter thermautotrophicus (MT-ACS1) and Archaeoglobus fulgidus (AFACS2). MT-ACS1 is a typical ACS in that acetate is the strongly preferred substrate over propionate and it cannot utilize larger substrates such as butyrate. Through modeling of MT-ACS1 on the S. enterica and yeast ACS structures, Ingram-Smith et al. (2006) identified four residues that comprise at least part of the acetate binding pocket of MT-ACS1 and have shown that alterations of these residues can greatly influence acyl substrate range and preference.

As observed for the $P$. aerophilum enzyme, AF-ACS2 is unusual in that it shows only a weak preference for acetate versus propionate and can also utilize butyrate and isobutyrate. The presence of the four acetate pocket residues in both AF-ACS2 and PA-ACS2 suggests that additional residues play an important role in determining substrate range and preference. The possible molecular basis for the broad substrate specificity of these two enzymes relative to MT-ACS1 and other characterized ACSs is discussed.

\section{Materials and methods}

Sequence and phylogenetic analysis

Putative ACS amino acid sequences were identified in BLASTP and TBLASTN searches (Altschul et al. 1990, 1997) of the finished genome sequences at the National Center for Biotechnology Information (NCBI) using the M. thermautotrophicus Z245 MT-ACS1 deduced amino acid sequence as the query. Acetyl-CoA synthetase sequences were aligned by Clustal X (Thompson et al. 1997) using a Gonnet PAM 250 weight matrix with a gap opening penalty of 10.0 and a gap ex- tension penalty of 0.05 . Aligned sequences were analyzed with the MEGA program (Kumar et al. 1994) using a neighbor joining algorithm with a gamma distance estimation $(\gamma=2)$. The phylogeny was constructed based on pairwise distance estimates of the expected number of amino acid replacements per site ( 0.2 in the scale bar). One thousand bootstrap replicates were performed and values of $80 \%$ or higher are shown. Sequences from only one strain or species of closely related bacteria were included in the analysis for brevity and readability.

Sequence similarity and identity were determined using the BLAST2 pairwise alignment program (http://www.ncbi.nlm.nih.gov/blast/bl2seq/wblast2.cgi). ConSurf (Armon et al. 2001, Glaser et al. 2003, Landau et al. 2005) (http://consurf.tau.ac.il) was used to determine evolutionary conserved residues that are likely to be important for protein structure and function.

\section{Cloning and sequencing the acs genes}

The genes encoding MT-ACS1 and MT-ACS2 from $M$. thermautotrophicus strains $\Delta \mathrm{H}, \mathrm{Z} 245$, and FTF, and AF-ACS2 from A. fulgidus were PCR amplified from genomic DNA using the following primer pairs: MT-ACS1, 5'-ATGTCAAAGGATACCTCAGTTCTCC-3' and 5'-CATCAAATATGAAGGGAGGGTATGG-3'; MT-ACS2, 5' ${ }^{\prime}$-ATGAGAGGACAGCTTGATGCTCTG-3' and 5'-CTGATTCTCCCATCGGCAAATGG-3'; AF-ACS2, 5' - ATGGCAGACCCGATGGAAGCTATG-3' and 5'-CCACTTTGAAGCCATACTACCACC- $3^{\prime}$. The PCR products were purified from agarose gel using the SpinPrep Gel DNA Kit (Novagen, Madison, WI). The PCR products were cloned into the pETBlue-1 expression vector (Novagen). The sequences of the cloned acs genes were confirmed by Li-Cor bidirectional sequencing at the Nucleic Acid Facility at Clemson University.

\section{Heterologous enzyme production in Escherichia coli}

The enzymes MT-ACS1, MT-ACS2, and AF-ACS2 were heterologously produced in E. coli Rosetta Blue(DE3). Cultures were grown at $37{ }^{\circ} \mathrm{C}$ in LB medium containing $50 \mu \mathrm{g}$ $\mathrm{ml}^{-1}$ ampicillin and $34 \mu \mathrm{g} \mathrm{ml}{ }^{-1}$ chloramphenicol to $A_{600}=0.6$. Heterologous protein production was induced by the addition of $0.5 \mathrm{mM}$ IPTG. Cells were grown overnight at $22-25^{\circ} \mathrm{C}$ and harvested.

\section{Enzyme purification}

A similar purification scheme was used for both MT-ACS1 and AF-ACS2. Cells suspended in ice-cold buffer A (25 mM Tris ( $\mathrm{pH}$ 7.5)) were disrupted by two passages through a French pressure cell at $138 \mathrm{MPa}$ and the cell lysate was clarified by ultracentrifugation. The supernatant was applied to a Q-sepharose fast-flow anion exchange column (GE Healthcare, Piscataway, NJ) that was developed with a linear gradient from 0 to $1 \mathrm{M} \mathrm{KCl}$ in buffer A. Fractions containing active enzyme were pooled and diluted with 0.5 volumes of buffer B (25 mM Tris (pH 7.0)) containing $2 \mathrm{M}$ ammonium sulfate and applied to a phenyl sepharose fast-flow hydrophobic interac- 
tion column (GE Healthcare) that was developed with a gradient from 0.7 to $0 \mathrm{M}$ ammonium sulfate in buffer $\mathrm{B}$. The purified enzymes were dialyzed against buffer B and concentrated to $>1 \mathrm{mg} \mathrm{ml}^{-1}$. The enzymes were purified to apparent homogeneity as judged by sodium dodecyl sulfate polyacrylamide gel electrophoresis (SDS-PAGE) (Laemmli 1970). Aliquots of the purified protein were stored at $-20{ }^{\circ} \mathrm{C}$. Protein concentrations were determined by the Bradford method (Bradford 1976) with bovine serum albumin as the standard.

\section{Molecular mass determination}

The native molecular mass of each enzyme was determined by gel filtration chromatography on a Superose 12 column (GE Healthcare) calibrated with chymotrypsinogen (25 kDa), ovalbumin (43 kDa), albumin $(67 \mathrm{kDa})$, aldolase $(158 \mathrm{kDa})$, catalase $(232 \mathrm{kDa})$, ferritin $(440 \mathrm{kDa})$ and blue dextran $(2000 \mathrm{kDa})$. Protein samples $(0.2 \mathrm{ml})$ were loaded onto the column pre-equilibrated with $50 \mathrm{mM}$ Tris ( $\mathrm{pH} 7.5$ ) containing $150 \mathrm{mM} \mathrm{KCl}$ and the column was developed at a flow rate of $0.5 \mathrm{mlmin}^{-1}$. The subunit molecular mass of each enzyme was determined by SDS-PAGE and was in agreement with the predicted size based on the deduced amino acid sequence.

\section{Enzymatic assays for ACS activity}

Enzymatic activity was determined by monitoring acetyl-CoA formation from acetate, ATP and CoA by the hydroxamate reaction (Lipmann and Tuttle 1945, Rose et al. 1954), in which activated acyl groups are converted to an acyl-hydroxamate and subsequently to a ferric hydroxamate complex that can be detected spectrophotometrically at $540 \mathrm{~nm}$. Reaction mixtures contained $100 \mathrm{mM}$ Tris ( $\mathrm{pH} 7.5), 600 \mathrm{mM}$ hydroxylamine$\mathrm{HCl}$ ( $\mathrm{pH}$ 7.0) and $2 \mathrm{mM}$ glutathione (reduced form), with varied concentrations of acyl substrate, $\mathrm{HSCoA}$, and $\mathrm{MgCl}_{2}$-ATP. A standard reaction temperature of $65^{\circ} \mathrm{C}$ was used, as this was determined to be the optimal temperature for both enzymes. Reactions were terminated by the addition of two volumes of stop solution (1 N HCl, $5 \%$ trichloroacetic acid, $1.25 \% \mathrm{FeCl}_{2}$ ). Acetyl-CoA formation was quantified by comparison with a standard curve prepared using known concentrations of acetyl-CoA in the reaction mixture. Reaction times for each enzyme were empirically determined such that the rate of the reaction remained linear and was within the acetyl-CoA standard curve.

For determination of apparent kinetic parameters, the concentration of one substrate (acyl substrate, HSCoA or equimolar MgATP) was varied and the other two substrates were held constant at saturating concentrations as follows: MT-ACS1, $40 \mathrm{mM}$ acetate, $20 \mathrm{mM} \mathrm{MgATP}, 0.5 \mathrm{mM}$ CoA; AF-ACS2, $50 \mathrm{mM}$ acetate, $20 \mathrm{mM}$ MgATP, $1 \mathrm{mM}$ CoA. Concentrations for the varied substrate generally ranged from 0.2 to 5-10 times the $K_{\mathrm{m}}$ value. For determination of apparent kinetic parameters for metals, the ATP concentration was held at $20 \mathrm{mM}$ for both enzymes and the metal concentrations were varied from 0.1 to $10 \mathrm{mM}$.

The apparent steady-state kinetic parameters $k_{\text {cat }}$ and $k_{\text {cat }} / K_{\mathrm{m}}$ and their standard errors were determined by nonlinear regres- sion to fit the data to the Michaelis-Menten equation using Kaleidagraph (Synergy Software). The enzymes followed Michaelis-Menten kinetics for all substrates with the exception that inhibition was observed above $0.5 \mathrm{mM} \mathrm{HSCoA}$ for MT-ACS1, in which case the kinetic parameters for acetate and ATP were performed in the presence of $0.5 \mathrm{mM} \mathrm{HSCoA}$ in the reaction mixture.

\section{Modeling the MT-ACS1 and AF-ACS2 structures}

The M. thermautotrophicus MT-ACS1 and the A. fulgidus AF-ACS2 structures were modeled on the S. enterica ACS structure (PDB ID: 1PG4) using DS Modeler (Accelrys Inc., San Diego, CA) and the default parameters. The structures were visualized with DS Visualizer (Accelrys) and DS Viewer Pro 5.0 (Accelrys). The models were visually compared with the $S$. enterica ACS structure to ensure there were no major structural anomalies.

\section{Genbank accession numbers}

Methanothermobacter thermautotrophicus $\triangle \mathrm{H}$ ACS1 (MTH217-MTH216), NP_275360 and NP_275359; and M. thermautotrophicus $\triangle \mathrm{H}$ ACS2 (MTH1603-MTH1604), NP_276715 and NP_276716. M. thermautotrophicus Z245 ACS1, DQ274062; and M. thermautotrophicus Z245 ACS2, DQ355203. M. thermautotrophicus FTF ACS1, DQ355204; and M. thermautotrophicus FTF ACS2, DQ355205; and A. fulgidus ACS2, NP_069202.

\section{Results \\ Presence of two ACS open reading frames (ORFs) in $M$. thermautotrophicus}

Analysis of the genome sequence of $M$. thermautotrophicus $\Delta \mathrm{H}$ revealed the presence of two putative ACSs, designated here as MT $\Delta \mathrm{H}-\mathrm{ACS} 1$ and MT $\Delta \mathrm{H}-\mathrm{ACS} 2$. The M. thermautotrophicus $\Delta H$ genome sequence annotation indicates the gene encoding MT $\Delta \mathrm{H}-\mathrm{ACS} 1$ is interrupted by a stop codon and a frame shift, resulting in two adjacent ORFs (MTH217MTH216, gi:2621263 and 2621262) that together have homology to full length ACS. The DNA region from the start ATG of MTH217 to the stop codon of MTH216 was amplified and cloned into the pETBlue-1 expression vector. A soluble truncated protein of about $63 \mathrm{kDa}$ was heterologously produced in E. coli but did not exhibit ACS activity (data not shown). This size is consistent with the position of the stop codon indicated in the published genome sequence. The sequence of the cloned $M$. thermautotrophicus $\Delta \mathrm{H}$ ACS1 gene (determined concurrently with the overexpression studies) confirmed the stop codon for MTH217 is authentic.

The genes encoding the ACS1 homologs from M. thermautotrophicus strains Z245 and FTF were also cloned for heterologous expression in E. coli. The three MT-ACS1 homologs share $98.5 \%$ amino acid sequence identity, with only nine positions that are not identical among all three (data not shown). Sequence analysis of the genes encoding the MT-ACS1 homologs from strains Z245 and FTF revealed the 
presence of a Glu codon at the equivalent position to the stop codon in the $M$. thermautotrophicus $\Delta \mathrm{H}$ MT-ACS1. Alteration of the stop codon in the gene encoding $M$. thermautotrophicus $\Delta \mathrm{H}$ MT-ACS1 to a Glu codon resulted in production of a full length, but insoluble, protein that was not characterized (data not shown).

The $M$. thermautotrophicus $\Delta H$ genome sequence annotation for the gene encoding MT-ACS2 indicates that this gene is also interrupted by a stop codon, as for MT-ACS1, resulting in two adjacent ORFs (MTH1603-MTH1604) that together have homology to full length ACS. The DNA region from the start ATG of MTH1603 to the stop codon of MTH1604 was amplified and cloned into the pETBlue-1 expression vector, as were the genes encoding the MT-ACS2 homologs from $M$. thermautotrophicus strains Z245 and FTF. The three MT-ACS2 homologs share $97 \%$ identity in deduced amino acid sequence, with only 12-14 amino acid differences between any two (data not shown). Sequence analysis of the cloned M. thermautotrophicus $\Delta H$ MT-ACS2 gene indicated an error in the $M$. thermautotrophicus $\Delta H$ genome sequence. An additional nucleotide is present that shifts the reading frame such that a full length protein of $73 \mathrm{kDa}$ is encoded.

Heterologous expression of the M. thermautotrophicus Z245 and FTF MT-ACS1 genes in E. coli resulted in soluble, active proteins of the expected size for a full length ACS. The M. thermautotrophicus Z245 MT-ACS1 (henceforth referred to as MT-ACS1) was purified to electrophoretic homogeneity and subjected to biochemical and kinetic characterization. Expression of each of the three MT-ACS2 genes in E. coli resulted in production of a $73 \mathrm{kDa}$ protein (data not shown), in agreement with the size predicted for the full length ACS. However, all three MT-ACS2 proteins were insoluble and were not characterized.

\section{Phylogenetic analysis of ACS}

Phylogenetic analysis of putative short and medium chain acyl-CoA synthetase sequences revealed several distinct clades, one of which contains all of the proven ACSs. Propionyl-CoA synthetase (Horswill and Escalante-Semerena 2002), Sa (Fujino et al. 2001b) and MACS1 (Fujino et al. 2001) acyl-CoA synthetases that show a preference for propionate, isobutyrate and octanoate, respectively, reside in other clades outside of the ACS clade. Within the ACS clade, shown in Figure 1, the sequences form eight major groups. Most ACS sequences group according to domain. Groups II and III are composed solely of eukaryotic sequences, with the exception of a single bacterial sequence in each. There are three large groups (I, IV and V) composed exclusively of bacterial sequences and several small bacterial clusters. The archaeal sequences fall into three groups (VI, VII and VIII), each of which also contains one or more bacterial sequences (Figure 1).
The S. enterica ACS and S. cerevisiae ACS1 sequences, representing the only two ACSs whose structures have been solved (Gulick et al. 2003, Jogl and Tong 2004), reside in Groups I and II. Among the putative archaeal ACSs, the sequences of the Haloarcula marismortui ACS1 and P. aerophilum ACS (PA-ACS), for which enzymatic activities have been proven (Brasen and Schonheit 2005, Brasen et al. 2005), reside in Groups VII and VIII, respectively (Figure 1). The Methanosaeta concilii ACS sequence in Group VII is identical to that of the Methanothrix soehngenii ACS, which has been purified and characterized (Jetten et al. 1989, Eggen et al. 1991).

To obtain a more thorough representation of the characteristics of archaeal enzymes across the ACS phylogeny, the M. thermautotrophicus MT-ACS1 and A. fulgidus AF-ACS2, whose sequences reside in groups VII and VIII of the phylogeny (Figure 1), were biochemically and kinetically characterized.

\section{Biochemical analysis of MT-ACS1 and AF-ACS2}

The enzymes MT-ACS1 and AF-ACS2 were heterologously produced in E. coli as soluble, active proteins and purified. The calculated masses for the MT-ACS1 and AF-ACS2 monomers are 71,556 $\mathrm{Da}$ and 77,587 Da, respectively. The molecular masses of the native enzymes were determined by gel filtration chromatography to be $144.8 \mathrm{kDa}$ and $221.2 \mathrm{kDa}$, respectively, suggesting MT-ACS1 is a dimer and AF-ACS2 is a trimer. The temperature optimum was determined to be about $65-70{ }^{\circ} \mathrm{C}$ for each enzyme (Figure 2). Less than $50 \%$ activity was observed below $45^{\circ} \mathrm{C}$ for both enzymes. At $80{ }^{\circ} \mathrm{C}$, MT-ACS 1 retained only $35 \%$ activity, whereas AF-ACS2 retained $44 \%$ activity at $90{ }^{\circ} \mathrm{C}$ and still had $20 \%$ activity at $100{ }^{\circ} \mathrm{C}$.

\section{Kinetic analysis of MT-ACS1 and AF-ACS2}

The acyl substrate range for MT-ACS1 was limited to acetate and propionate; the enzyme was unable to utilize butyrate. However, AF-ACS2 was able to utilize acetate, propionate and butyrate. This enzyme also had strong, but unsaturable, activity with isobutyrate and weak, but unsaturable, activity with valerate, but could not utilize larger acyl substrates or other branched chain acyl substrates. The kinetic parameters determined for each enzyme are shown in Table 1.

There are a number of noteworthy points to be made from the results in Table 1 . The two enzymes have similar affinity $\left(K_{\mathrm{m}}\right)$ for acetate but very different affinities for propionate. The affinity of AF-ACS2 for propionate is almost identical to that of MT-ACS1 for acetate and nearly tenfold higher than the affinity of MT-ACS1 for propionate. Whereas the difference in affinity between the two substrates is over tenfold for MT-ACS1, AF-ACS2 has only a 2.3-fold higher affinity for acetate than propionate. MT-ACS1 has a strong preference for

Figure 1 (facing and following page). Phylogeny of ACS sequences. A phylogeny of putative short and medium chain acyl-CoA synthetases from the finished genome sequences available at NCBI was constructed using the neighbor joining algorithm of MEGA (Kumar et al. 1994). Only the major clade containing the proven ACSs is shown here. For most genera, sequences from only one species were used in constructing the phylogeny for brevity and readability. Eukaryotic sequences are indicated in black, bacterial sequences in red and archaeal sequences in blue. 


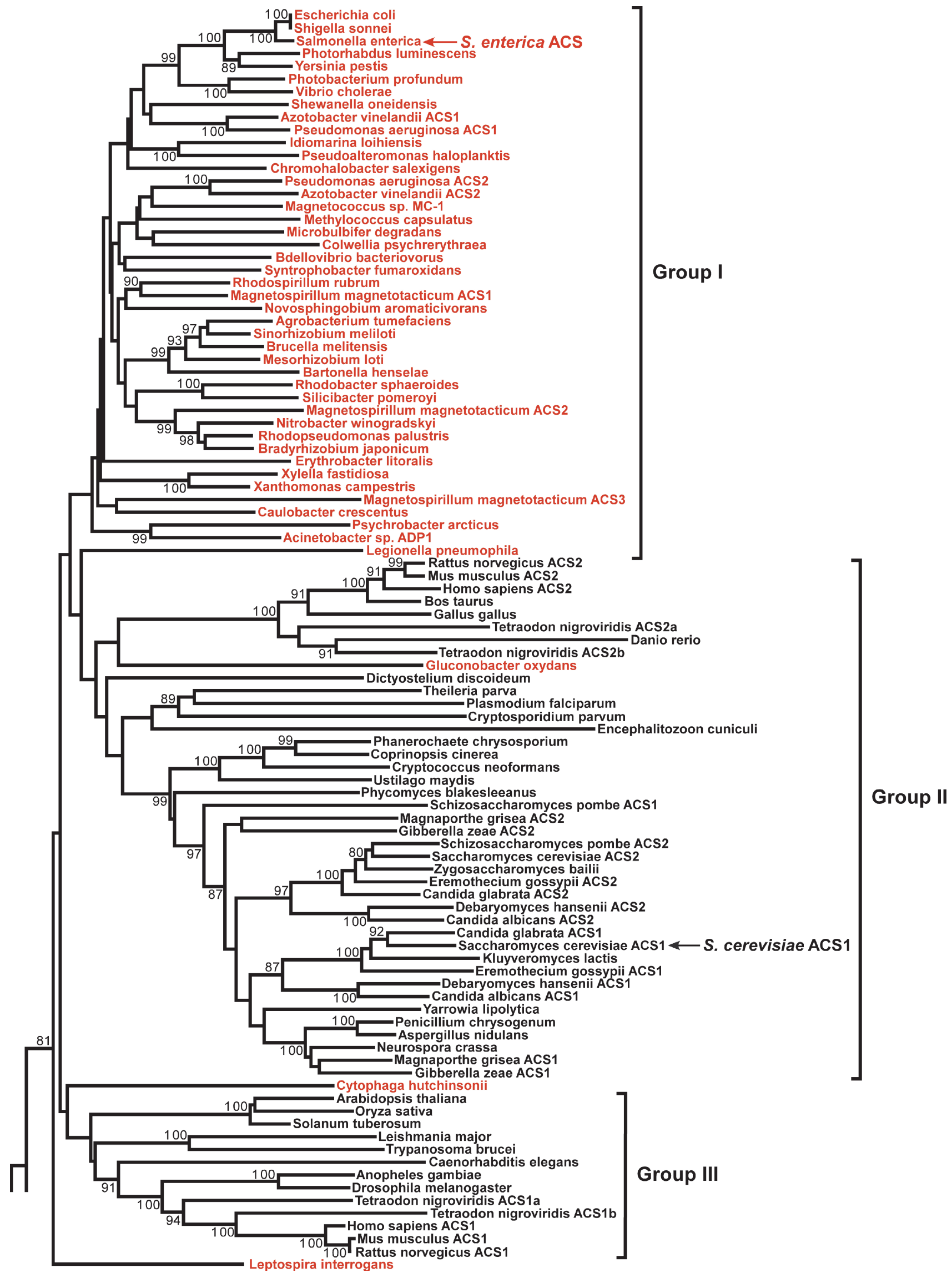




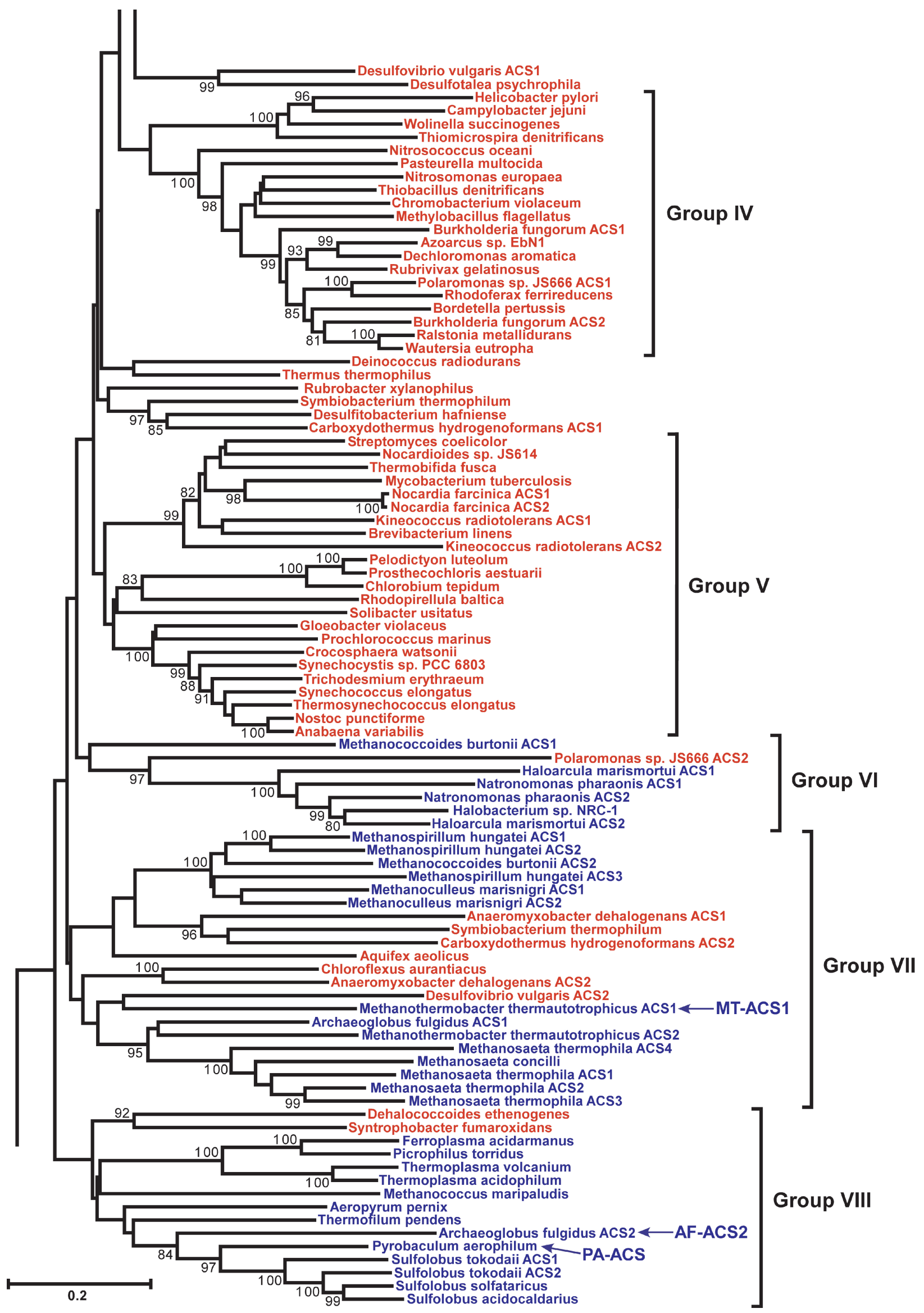




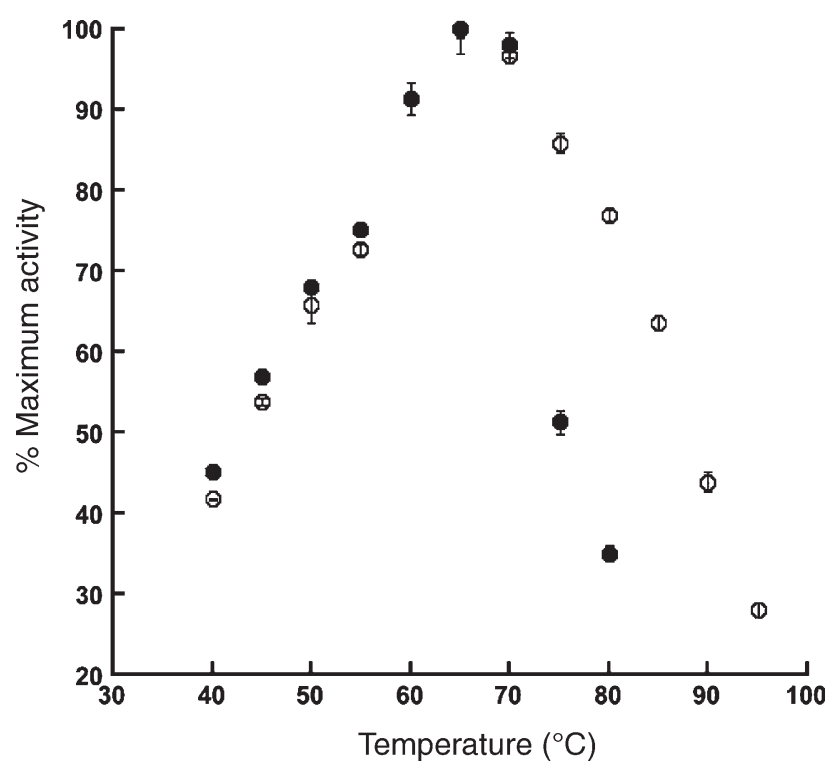

Figure 2. Temperature optima for MT1-ACS and AF-ACS2. Enzyme reactions were performed at the indicated temperatures in triplicate. Activities are reported as a percentage of the maximum activity determined for each enzyme. Symbols: $\bigcirc=$ MT1-ACS; and $\bigcirc=$ AFACS2.

acetate as the substrate as shown by the 14-fold higher catalytic efficiency $\left(k_{\text {cat }} / K_{\mathrm{m}}\right)$ with acetate versus propionate. However, AF-ACS2 has only a 2.3-fold higher preference for acetate over propionate. Finally, AF-ACS2 was able to utilize butyrate as substrate, although the $K_{\mathrm{m}}$ was 78 -fold higher than that for acetate and 34-fold higher than that for propionate, and the turnover rate was 21-fold reduced with butyrate compared with acetate or propionate.

The $K_{\mathrm{m}}$ values for CoA for both enzymes showed less than twofold difference (Table 1), and the $K_{\mathrm{m}}$ values for ATP were

Table 1. Kinetic parameters for MT-ACS1 and AF-ACS2.

\begin{tabular}{llcll}
\hline Substrate & $\begin{array}{l}\text { Enzyme } \\
(\mathrm{mM})\end{array}$ & $\begin{array}{l}K_{\mathrm{m}} \\
\left(\mathrm{s}^{-1}\right)\end{array}$ & $k_{\text {cat }}$ & $\begin{array}{l}k_{\text {cat }} / K_{\mathrm{m}} \\
\left(\mathrm{s}^{-1} \mathrm{mM}^{-1}\right)\end{array}$ \\
\hline Acetate & MT-ACS1 $^{1}$ & $3.5 \pm 0.1$ & $65.4 \pm 0.3$ & $18.6 \pm 0.5$ \\
& AF-ACS2 $^{1}$ & $1.7 \pm 0.06$ & $35.9 \pm 0.58$ & $21.2 \pm 0.4$ \\
Propionate & MT-ACS1 & $36.5 \pm 1.9$ & $46.3 \pm 0.7$ & $1.3 \pm 0.04$ \\
& AF-ACS2 $^{1}$ & $3.9 \pm 0.02$ & $35.7 \pm 0.17$ & $9.1 \pm 0.06$ \\
Butyrate & MT-ACS1 $^{1}$ & - & - & - \\
& AF-ACS2 & $133.0 \pm 14.6$ & $1.68 \pm 0.07$ & $0.013 \pm 0.0009$ \\
Valerate & MT-ACS1 & - & - & - \\
& AF-ACS2 & Unsaturable & & \\
Isobutyrate & MT-ACS1 & - & - & - \\
ATP & AF-ACS2 & Unsaturable & & \\
& MT-ACS1 & $3.3 \pm 0.2$ & $66.6 \pm 0.9$ & $20.2 \pm 0.9$ \\
CoA & AF-ACS2 & $2.9 \pm 0.01$ & $38.1 \pm 0.12$ & $13.2 \pm 0.09$ \\
& MT-ACS1 & $0.19 \pm 0.003$ & $81.6 \pm 0.7$ & $423.7 \pm 4.7$ \\
& AF-ACS2 & $0.30 \pm 0.003$ & $44.3 \pm 0.2$ & $144.9 \pm 1.2$ \\
\hline
\end{tabular}

\footnotetext{
${ }^{1}$ Kinetic parameters for MT-ACS1 were determined in the presence of $0.5 \mathrm{mM} \mathrm{CoA}$.
}

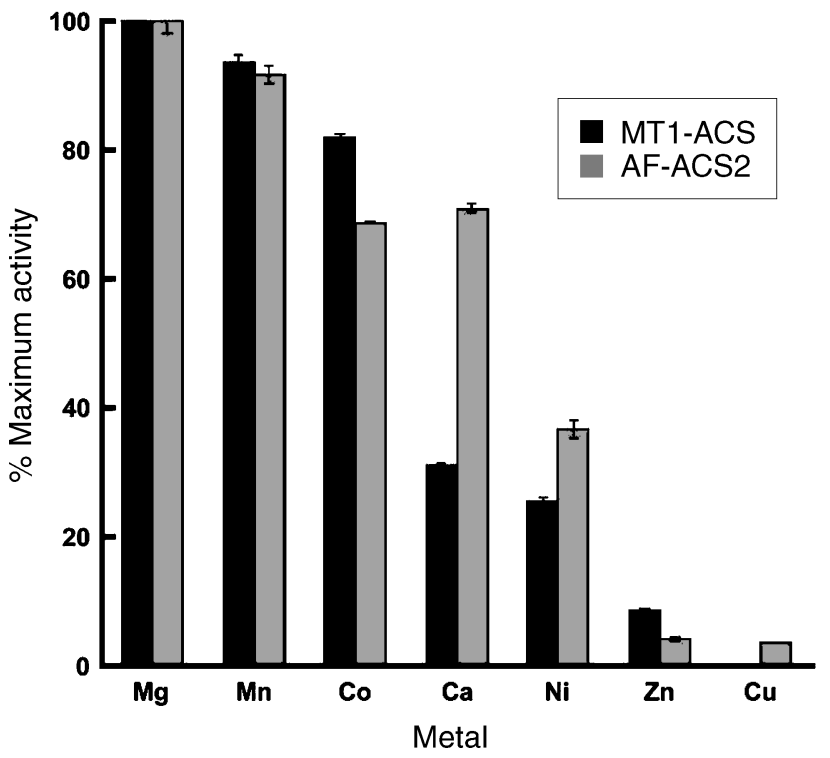

Figure 3. Divalent metal specificity for MT1-ACS and AF-ACS2. Enzyme reactions were performed in triplicate at $65^{\circ} \mathrm{C}$ in the presence of $20 \mathrm{mM}$ metal (as the chloride salt) $+20 \mathrm{mM}$ ATP. Activities are reported as a percentage of the maximum activity determined for each enzyme with $\mathrm{Mg}^{2+}$ as the metal substrate.

similar for both enzymes. Both enzymes demonstrated a strong preference for ATP versus CTP, GTP, TTP, UTP, ITP or ADP, for which less than $5 \%$ activity was observed (data not shown).

The metal specificity was tested for each enzyme using a standard metal concentration of $20 \mathrm{mM}$ and $20 \mathrm{mM}$ ATP. Both MT-ACS1 and AF-ACS2 showed strong preference for $\mathrm{Mg}^{2+}$ and $\mathrm{Mn}^{2+}$ as the divalent metal (Figure 3) and $\mathrm{Co}^{2+}$ also gave high activity. Strong activity was observed with $\mathrm{Ca}^{2+}$ for AF-ACS2 but not for MT-ACS1. Moderate activity was observed for both enzymes with $\mathrm{Ni}^{2+}$, whereas $\mathrm{Cu}^{2+}$ and $\mathrm{Zn}^{2+}$ worked poorly for both enzymes. The kinetic parameters determined for those metals that gave the highest activity are shown in Table 2. For MT-ACS1, the highest affinity and turnover rate were observed with $\mathrm{Mg}^{2+}$. Although the highest turnover rate was observed with $\mathrm{Mg}^{2+}, \mathrm{Mn}^{2+}$ and $\mathrm{Ca}^{2+}$ gave the highest catalytic efficiencies for AF-ACS2.

\section{Discussion}

Although our kinetic and biochemical characterization of MT-ACS1 and AF-ACS2 expands our knowledge of the properties of ACSs, there is still a paucity of information on this important class of enzymes. With the advent of whole genome sequencing, gene functions are usually assigned based on homology with other sequences in the sequence databases. In many cases, a particular enzymatic function may be assigned based on homology to just a single sequence whose function has been proven. Acetyl-CoA synthetase is widespread in all three domains, and most putative ACSs have been assigned this function through homology. Of the 193 ACS sequences 
Table 2. Kinetic parameters for divalent metals for MTI-ACS and AF-ACS2.

\begin{tabular}{|c|c|c|c|c|}
\hline Enzyme & Metal & $\begin{array}{l}K_{\mathrm{m}} \\
(\mathrm{mM})\end{array}$ & $\begin{array}{l}k_{\text {cat }} \\
\left(\mathrm{s}^{-1}\right)\end{array}$ & $\begin{array}{l}k_{\text {cat }} / K_{\mathrm{m}} \\
\left(\mathrm{s}^{-1} \mathrm{mM}^{-1}\right)\end{array}$ \\
\hline \multirow[t]{3}{*}{ MT1-ACS ${ }^{1}$} & $\mathrm{Mg}^{2+}$ & $0.38 \pm 0.02$ & $56.5 \pm 1.0$ & $147.5 \pm 4.5$ \\
\hline & $\mathrm{Mn}^{2+}$ & $0.61 \pm 0.02$ & $44.6 \pm 0.5$ & $72.8 \pm 2.3$ \\
\hline & $\mathrm{Co}^{2+}$ & $0.99 \pm 0.03$ & $39.4 \pm 0.3$ & $40.0 \pm 1.1$ \\
\hline \multirow[t]{4}{*}{ AF-ACS2 } & $\mathrm{Mg}^{2+}$ & $1.08 \pm 0.05$ & $23.4 \pm 0.3$ & $21.8 \pm 0.7$ \\
\hline & $\mathrm{Mn}^{2+}$ & $0.70 \pm 0.02$ & $20.6 \pm 0.23$ & $29.7 \pm 0.76$ \\
\hline & $\mathrm{Ca}^{2+}$ & $0.64 \pm 0.006$ & $18.2 \pm 0.05$ & $28.3 \pm 0.27$ \\
\hline & $\mathrm{Co}^{2+}$ & $1.50 \pm 0.02$ & $19.0 \pm 0.13$ & $12.2 \pm 0.09$ \\
\hline
\end{tabular}

${ }^{1}$ Kinetic parameters for MT-ACS1 were determined in the presence of $0.5 \mathrm{mM} \mathrm{CoA}$.

shown in the phylogeny in Figure 1, only a handful have been biochemically characterized. The $S$. enterica ACS and $S$. cerevisiae ACS1, whose structures have been solved (Gulick et al. 2003, Jogl and Tong 2004), are quite distant from the archaeal ACSs, for which there is no structure.

Although genes predicted to encode for ACS are widespread in the Archaea, only a few archaeal ACSs have been biochemically characterized. Acetyl-CoA synthetase activity was first detected in archaea in Methanothermobacter marburgensis (formerly Methanobacterium thermoautotrophicum Marburg) (Oberlies et al. 1980), a thermophilic chemolithoautotrophic methanoarchaeon that can utilize $\mathrm{H}_{2} / \mathrm{CO}_{2}$ as the sole carbon and energy source (Zeikus and Wolfe 1972). When M. marburgensis (closely related to M. thermautotrophicus) was grown on $\mathrm{H}_{2} / \mathrm{CO}_{2}$ in the presence of acetate, $10 \%$ of cellular carbon was derived from acetate with the remainder derived from $\mathrm{CO}_{2}$ (Fuchs et al. 1978). Oberlies et al. (1980) subsequently demonstrated ACS activity in $M$. marburgensis cells grown with limiting $\mathrm{H}_{2} / \mathrm{CO}_{2}$ and proposed that ACS allows assimilation of acetate as a cellular carbon source in order to spare limited supplies of $\mathrm{CO}_{2}$.

The first archaeal ACSs purified and characterized were those from M. soehngenii and Methanothrix thermophila CALS-1 (now Methanosaeta concilii and Methanosaeta thermophila CALS-1) (Jetten et al. 1989, Teh and Zinder 1992). Acetyl-CoA synthetase is the first enzyme in the activation of acetate to acetyl-CoA for methanogenesis in the obligately acetoclastic Methanosaeta (Jetten et al. 1989, Teh and Zinder 1992, Allen and Zinder 1996). In fact, the recently completed genome sequences of Methanosaeta species reveal that $M$. thermophila $\mathrm{P}_{\mathrm{T}}$ has four genes that encode ACS and M. concilii has five (K.S. Smith and C. Ingram-Smith, unpublished data). All of the methanoarchaea with ACS have at least two acs genes, with the exception of Methanococcus maripaludis (Figure 1).

A number of halophilic archaea are able to utilize acetate as a carbon and energy source. Brasen and Schonheit (Brasen and Schonheit 2001, Brasen and Schonheit 2004) demonstrated that Halococcus saccharolyticus, Haloferax volcanii, Halorubrum saccharovorum and $H$. marismortui grown on acetate as a carbon and energy source exhibited ACS activity.
During growth on glucose, these halophiles excreted acetate into the media and exhibited ADP-forming acetyl-CoA synthetase activity (ADP-ACS; acetyl-CoA + ADP $+\mathrm{P}_{\mathrm{i}} \leftrightarrow$ acetate + ATP + CoA) but not ACS activity. Upon entry into stationary phase, ACS activity was induced and the excreted acetate was consumed. Thus, ADP-ACS was determined to be responsible for acetate and ATP production from excess acetyl-CoA during growth on glucose but ACS was responsible for activation of acetate for use as a carbon and energy source.

Although the H. marismortui, M. soehngenii, and M. thermophila CALS-1 ACSs and the M. thermautotrophicus MT-ACS1 all show a strong preference for acetate, the characterized ACSs from A. fulgidus and P. aerophilum have an expanded substrate range. What physiological purpose could this broad substrate range serve? One possibility is that $A$. fulgidus and $P$. aerophilum can utilize a more diverse array of carbon or energy sources than other archaea. Both P. aerophilum and A. fulgidus can utilize complex organics such as yeast extract, meat extract, tryptone, and peptone as growth substrates (Stetter 1988, Volkl et al. 1993), whereas the others cannot. The presence of an ACS with an expanded substrate range may provide a means for utilization of other short chain fatty acids either scavenged from the environment or from the breakdown of complex organics without the need for additional enzymes.

The findings of the phylogenetic analysis presented here (Figure 1) contrast with those of Brasen and Schonheit (2005) with respect to the archaeal ACSs. In their analysis, the archaeal sequences formed one distinct clade, leading to the conclusion that the archaeal sequences form a separate branch within the prokaryotic sequences and have a monophyletic origin (Brasen and Schonheit 2005). In our analysis, the archaeal sequences form three groups, each of which also contains bacterial sequences. This may be a result of the larger number of sequences from each domain used in this analysis (193 sequences composed of 107 bacterial, 33 archaeal, and 53 eukaryotic sequences versus 51 total sequences by Brasen and Schonheit (2005)) and differences in methodology. However, a separate phylogenetic analysis using the minimum evolution algorithm of the MEGA package (Kumar et al. 1994) showed a similar result (data not shown).

Overall, the ACSs show strong sequence conservation regardless of domain, as indicated by pairwise amino acid sequence comparisons that show MT-ACS1 and AF-ACS2 share $38-49 \%$ identity and $57-67 \%$ similarity with $S$. cerevisiae ACS1 and S. enterica ACS. However, the subunit composition of the ACSs is quite diverse, with monomeric (S. enterica and H. marismortui ACSs (Brasen and Schonheit 2005, Gulick et al. 2003), dimeric (MT-ACS1, M. concilii, and Bradyrhizobium japonicum ACSs (Jetten et al. 1989, Preston et al. 1990, Lee et al. 2001)), trimeric (AF-ACS2 and S. cerevisiae ACS1 (Jogl and Tong 2004)), and octameric enzymes (PA-ACS (Brasen et al. 2005)) thus far characterized.

Characterized enzymes from groups I, II, III, VI, and VII, which include the bacterial ACSs from S. enterica (Gulick et al. 2003) and B. japonicum (Preston et al. 1990, Lee et al. 2001), the eukaryotic ACS1 and ACS2 enzymes from human 
(Luong et al. 2000, Fujino et al. 2001a) and S. cerevisiae (van den Berg et al. 1996), and archaeal ACSs from M. concilii (M. soehngenii) (Jetten et al. 1989, Eggen et al. 1991), H. marismortui (Brasen and Schonheit 2005), and M. thermautotrophicum (MT-ACS1), all show a strong preference for acetate as the acyl substrate, with propionate being the only alternative acyl substrate. AF-ACS2 and the P. aerophilum PA-ACS (Brasen et al. 2005), both from group VIII, have an expanded substrate range that includes butyrate and the branched chain isobutyrate as well as acetate and propionate. AF-ACS2 shows only a weak (twofold) preference for acetate over propionate, unlike most ACSs that generally have a 10- to 20 -fold preference for acetate, as determined by the higher catalytic efficiency $\left(k_{\text {cat }} / K_{\mathrm{m}}\right)$.

Although a full kinetic characterization of PA-ACS was not reported, the $K_{\mathrm{m}}$ value for acetate was determined to be $3 \mu \mathrm{M}$, over 500-fold lower than that observed for AF-ACS2. Although this may indicate that these enzymes have very different kinetic properties, it may also be due to differences in the enzyme assay used in the two studies. The $K_{\mathrm{m}}$ value for acetate observed for PA-ACS is at least 20- to several hundredfold lower than that observed for any other ACS. The $K_{\mathrm{m}}$ value for acetate for AF-ACS2 is well in line with the values determined using the hydroxamate assay with other archaeal ACSs including MT-ACS1 (Jetten et al. 1989, Preston et al. 1990, Teh and Zinder 1992). It is not known whether these differences are meaningful with regard to whether these enzymes may represent a subgroup of ACSs that show only weak preference for acetate and expanded substrate range.

These findings lead one to question whether AF-ACS2 and PA-ACS are anomalies within the ACSs or whether they represent a subset of enzymes with different properties from the "traditional" ACSs that have a strong preference for acetate and a narrow substrate range. Four residues (Ile ${ }^{312}, \mathrm{Thr}^{313}$, $\mathrm{Val}^{388}$, and $\operatorname{Trp}^{416}$ ) have been shown to form the acetate binding pocket of MT-ACS1 and have been shown to be important in acyl substrate selection (Ingram-Smith et al. 2006). Alteration of any of these four residues influences substrate affinity or substrate range, or both, as well as catalysis (Ingram-Smith et al. 2006). For example, alteration of $\operatorname{Trp}^{416}$ to Gly, the residue found in short and medium chain acyl-CoA synthetases other than acetyl- and propionyl-CoA synthetases, expands the substrate range of MT-ACS1 such that the enzyme can utilize substrates ranging from acetate to octanoate (including some branched chain acyl substrates) and changes the substrate preference from acetate to valerate. In propionyl-CoA synthetase, $\mathrm{Val}^{388}$ is replaced by Ala. A Val ${ }^{388}$ Ala MT-ACS1 variant has higher affinity for propionate than acetate and a slightly greater preference for propionate as well. Among the ACS sequences in Figure 1, including both AF-ACS2 and PA-ACS, $\mathrm{Thr}^{313}, \mathrm{Val}^{388}$, and $\mathrm{Trp}^{416}$ are completely conserved and $\mathrm{Ile}^{312}$ is highly conserved, with Val as the only other amino acid observed at the equivalent position.

Within group VIII in the ACS phylogeny in Figure 1, a subclade consisting of AF-ACS2 and PA-ACS as well as four Sulfolobus sequences is strongly supported by bootstrapping $(84 \%)$. These sequences were aligned with the S. enterica
ACS, MT-ACS1, and H. marismortui ACS sequences and a partial alignment is shown in Figure 4. ConSurf analysis (Armon et al. 2001, Glaser et al. 2003, Landau et al. 2005) of the alignment was performed to help delineate amino acid residues that might contribute to the broad substrate range of AF-ACS2 and PA-ACS and suggest whether members of this subclade within group VIII represents a subclass of ACSs with expanded substrate range. ConSurf calculates evolutionary conservation scores for each residue within a protein sequence based on protein structure, multiple sequence alignment, evolutionary distance between sequences, and evolutionary tree topology. The evolutionary conservation scores are then mapped onto the protein structure to define probable regions of structural and functional importance.

Using the $S$. enterica ACS structure as the query for ConSurf analysis, the evolutionary conservation score for each position along with the alternative residues from the fifty most closely related sequences were determined. Salmonella enterica ACS residues determined to be the most highly conserved by ConSurf analysis are shaded in the partial alignment (Figure 4), along with residues in the other sequences that are identical or among the alternative residues for that position. In the complete alignment, eleven residues with high ConSurf scores (all of which are shown in the partial alignment in Figure 4) are conserved in the three ACS sequences representing enzymes with "traditional" characteristics, but differ in AF-ACS2 and PA-ACS. In addition, the four Sulfolobus sequences that reside in the same subclade in group VIII as AF-ACS2 and PA-ACS of the phylogeny also differ at these same positions.

MT-ACS1 and AF-ACS2 have been modeled on the S. enterica ACS structure to determine whether the acetate binding pockets show any major differences that could be attributed to the differences in substrate preference and substrate range of these two enzymes. The models depicted in Figure 5 show residues within $10 \AA$ of the propyl group of the adenosine- $5^{\prime}$-propylphosphate ligand. In the $S$. enterica structure, the adenosine- $5^{\prime}$-propylphosphate mimics the acetyladenylate intermediate and the propyl group approximates the position of acetate in the active site (Gulick et al. 2003). Overall, the acetate binding sites of the two modeled enzymes are very similar (Figure 5). However, there are key differences in the positioning of certain residues that are conserved in both enzymes and in residues that were identified by the ConSurf analysis (Figure 4) to be conserved in the traditional ACSs but not the AF-ACS2/PA-ACS subclade of group VIII (Figure 1).

The propyl group is in a similar position in both the MT-ACS1 and AF-ACS2 models, and three of the acetate pocket residues occupy similar positions as well. However, whereas $\mathrm{Ile}^{312}$ of MT-ACS points away from the propylphosphate group (Figure 5A), Ile ${ }^{329}$ of AF-ACS2 points inwards (Figure 5B). This may increase the hydrophobicity of the acetate pocket and could account in part for the higher affinity of AF-ACS2 for acyl substrates than observed for MT-ACS1. Among residues identical to both enzymes, the other major difference observed in the acetate pocket region is that $\mathrm{Leu}^{424}$ of MT-ACS1 is positioned quite differently from 
$312 / 313$

SE-ACS MT-ACS 1 $\mathrm{HM}-\mathrm{ACS}$

AF-ACS2

PA-ACS

ST-ACS1 ST-ACS2 ** 416 $* * * * * * *$
279 GGYLVYAATTFKYVFDYHPGDIYWCTADVGWVTGHSYLLYGPLACGATTLMFEGVPNWPT 338 281 GGYMVGVASTLEMTFDIHNGDLWWCTADIGWITGHSYVVYGPLLLGTTTLLYEGAPDYPD 340 288 GGYLAWAAWTSQAVLDIKPEDTYFCSADIGWITGHSYIVYGPLALGTTTMMYEGTPDYPD 347 298 GGYAVHLYTTMKLIFDIKDDDIFWCTADIGWITGHSYIVYGPLMCGATTMMYEGAPDFPD 357 294 GGWAVHVYATMKWVFDIRDDDIFWCTADIGWVTGHSYVVLGPLLMGATEVIYEGAPDYPQ 353 287 GGYLTILHATMNWVFDIKDSDIMWTTADIGWITGHSYIVFGPLLEGVTTVMYEGALDYPN 346 286 GGYLTLLHATMKWVFDVRDDDIYWCTADIGWVTGHSYIVFGPLMEGVTEIMYEGALDYPK 345 288 GGYMTLLHATMKWVFDIRDDDIYWCTADIGWVTGHSYIVFGPLMEGATEIMYEGALDYPQ 347 288 GGYETLLHATMRWVFDIRDDDIYWCTADIGWVTGHSYIVFGPLTEGATTVMYEGALDYPQ 347

339 PARMCQVVDKHQVNILYTAPTAIRALMAEGDKAIEGTDRSSLRILGSVGEPINPEAWEWY 398 341 PGVWWSIVEKYGVTKFYTAPTAIRHLMRFGDKHPKRYNLESLKILGTVGEPINPEAWMWY 400 348 KDRLWDIVEEYEADQLYTAPTAIRAFMKWGKQYPEQHDLSSLRLLGTVGEPINPRAWKWY 407 358 VSRWAAMIERYGVTIFYTAPTAIRMLMRFPEENYTKYDMSTLRI IHSVGEPINPEAWRWY 417 354 PDRWWSIIERYGVTIFYTSPTAIRMFMRYGEEWPRKHDLSTLRIIHSVGEP INPEAWRWA 413 347 PDRWASIIERYGVTILYTSPTAIRSEMKLGEDVYKGRDFSSVRLMHSVGEPINPEAFRWF 406 346 PDRWVSIIERYGVTIFYTSPTAIRSFMKYGDEWVKAHKTDTIRIIHSVGEPINPEAFEWL 405 348 PDRWVSIIERYGITILYTSPTAIRSEMKYGDNWVKAHITSTVRLMHSVGEPINPEAWEWL 407 348 PDRWVSIIERHSVTILYTSPTAIRTFMKFGEEWVKKHDTSTVRLIHSVGEPINPEAQDWM 407

399 WKKIGKEKCPVVDTWWQTETGGFMITPLPG---AIELKAGSATRPFFGVQPALVD-NEGH 454 401 YRNIGREKCPIIDTWWQTETGMHLIAPLPVT----PLKPGSVTKPLPGIEADVVD-ENGD 455 408 YKHIGNEECPVVDTWWQTETGGMMITTLPGV---KDMKPGSAGPPLPGNDVRIVD-TEGE 463 418 YRVFGREDVVCSSTWWMTETGGMLTDHFPGLGKIIPLKPGTNGYPIPGVRMEVLD-DEGN 476 414 YRVLGNEKVAFGSTWWMTETGGIVISHAPGL-YLVPMKPGTNGPPLPGFEVDVVD-ENGN 471 407 FRLVGKESIPFGSTWWMTETGGIMISHLPGL-YLIPLKPGTNGMPLPGIDADVVN-ENGN 464 406 WKLIGRGEVPFGSTWWMTETGGIMISHLPGL-YLIPLKPGTNGMPLPGIEADVVD-DNGN 463 408 WKLVGREEVPFGSTWWMTETGGILISHLPGL-YLVPMKPGTNGPPILGIEPDVVN-EDGK 465 408 WKLVGREEIPFGSTWWMTETGGIMISHLPGL-YLIPMKPGTNGMPIMGVEADVVN-DDGK 465

455 PQEG-ATEGNLVITDSWP-GQARTLFGDHERFEQTYFSTFKN- - - - - - - MYFSGDGAR 503 456 PVPL-GKGGFLVIRKPWP-AMFRTLFNDEQRYIDVYWKQIPGG- - - - - - - VYTAGDMAR 505 464 EVEP-GRAGYLTVDKPWP-GMLRTLYKNDERF IDEYWAEYSDTDSDDSDDWVYFPEDGAK 521 477 SVKP-GERGYLVISTPWP-GMLMTLFKDPNRFIDVYFGKYKSKG- - - - -WYYYTGDFAM 528 472 PAPP-GVKGYLVIKKPWP-GMLHGIWGDPERYIKTYWSRFPG- - - - - - -MFYAGDYAI 520 465 PTKP-EERGYLVIRKPWP-GMPLTIWGDPDRYVKVYWSKFPG- - - - - - - - IFYPGDFAV 513 464 PTKP-EERGYLVIKNPWP-GMPLTIHRDPERYIKIYWSKFPG- - - - - - - MFYAGDYAV 512 466 QVNL-EERGYLVIKNPWP-GMPLTIYKDPERYVKVYWSRFPG- - - - - - -MFYVGDYAV 514 466 PSNL-EDRGYLVIKNPWP-GMPLTIYKDPERYVKVYWSKFPG-

504 RDEDGYYWITGRVDDVLNVSGHRLGTAEIESALVAHPKIAEAAVVGIPHAIKGQAIYAYV 563 505 KDEDGYFWIQGRSDDVLNIAGHRIGTAEVESVFVAHPAVAEAAVIGKADPI KGEVIKAFL 565 522 IDDDGYITVLGRVDDVINVSGHRLGTMEIESAIVGVEGVAEAAVVGGDHDIKGEAVYAYV 581 529 FDEDGYVWVLGRADDILKVAGHRIGTAEIESAMVSHPDVAEAACVGKSDPVKGEIPIIYV 588 521 KDKDGYIWVLGRADEVIKVAGHRLGTYELESALISHPAVAESAVVGVPDAIKGEVPIAFV 580 514 KDSDGYFWILGRADEVIKVAGHRLGTYELESALIHHPAVAEAAVVGVPDPLKGEVPVAFV 573 513 KDSDGYFWVLGRADEVIKVAGHRLGTYELESAI IEHPAVAEAAVVGVPDPVKGEVPVAFV 572 515 KDKDGYFWILGRADEVIKVAGHRLGTYELESALIEHPAVAEAAVIGVPDPVKGEVPYAFV 574 515 KDRDGYFWILGRADEVIKVSGHRLGTYELESALIEHPAVAEAAVVGVPDPVKGEVPYAFV 574
Figure 4. Alignment and ConSurf analysis of ACS sequences. The $M$. thermautotrophicus MT-ACS1 and A. fulgidus AF-ACS2 amino acid sequences were aligned with the $S$. enterica ACS sequence (SE-ACS) and the ACS sequences from $P$. aerophilum (PA-ACS), Sulfolobus tokodaii (ST-ACS1 and ACS2), Sulfolobus solfataricus (SSACS), and Sulfolobus acidocaldarius (SA-ACS) using Clustal X (Thompson et al. 1997). A partial alignment is shown here. Residues of the S. enterica ACS found to have high evolutionary conservation scores by ConSurf analysis (Armon et al. 2001, Glaser et al. 2003, Landau et al. 2005) (http://consurf.tau.ac.il) are shaded, as are residues in the other ACS sequences that are identical or among the alternative residues listed for each of these highly conserved positions. Asterisks indicate those positions that are identical in all nine sequences. The acetate binding pocket residues are boldfaced and numbered above the aligned sequences according to their position within MT-ACS1. Those residues at positions with high ConSurf scores that differ in AF-ACS2, PA-ACS, and the Sulfolobus sequences from the three ACS sequences representing enzymes with "traditional" characteristics are indicated in red. the equivalent residue $\mathrm{Leu}^{441}$ of AF-ACS2. The overall increased hydrophobicity of the acyl substrate binding pocket of AF-ACS2 may positively influence substrate affinity, but it is likely that the combined effects of multiple residues are required to determine whether the enzyme has a narrow or broad substrate range.

Of the eleven residues identified by ConSurf analysis to be highly conserved in traditional ACSs but not the AFACS2/PA-ACS subclade (Figure 4), six of these positions are in close proximity to $\mathrm{Val}^{388}$ and $\operatorname{Trp}^{416}$ of MT-ACS1 (Figure 5A), the two acetate pocket residues that were shown to have the greatest influence on substrate range and preference (Ingram-Smith et al. 2006). Five of these residues $\left(\mathrm{Leu}^{385}, \mathrm{Gly}^{386}, \mathrm{Ile}^{412}, \mathrm{Asp}^{413}\right.$, and Pro ${ }^{427}$ of MT-ACS1 and
$\mathrm{Ile}^{402}$, $\mathrm{His}^{403}, \mathrm{Ser}^{429}, \mathrm{Ser}^{430}$, and His ${ }^{444}$ of AF-ACS2) are clustered to one side of the pocket for both enzymes (Figure 5) and may influence the positioning of $\mathrm{Glu}^{390}$, which points slightly inward toward the pocket in MT-ACS1, and the equivalent residue $\mathrm{Glu}^{407}$ of AF-ACS2 that points slightly away from the acetate pocket. Withdrawal of the negative charge of this residue from the acetate pocket in AF-ACS2 may positively influence substrate binding.

The sixth residue, represented by $\operatorname{Gl}^{417}$ of MT-ACS1 and $\mathrm{Met}^{434}$ of AF-ACS2, is more intimately positioned near the acetate pocket (Figure 5). Glutamine ${ }^{417}$ points away from the acetate pocket and the propylphosphate group, whereas $\mathrm{Met}^{434}$ bends in toward the propylphosphate. $\mathrm{Met}^{434}$ may play a role in expanding the substrate range of AF-ACS2 by extending the 

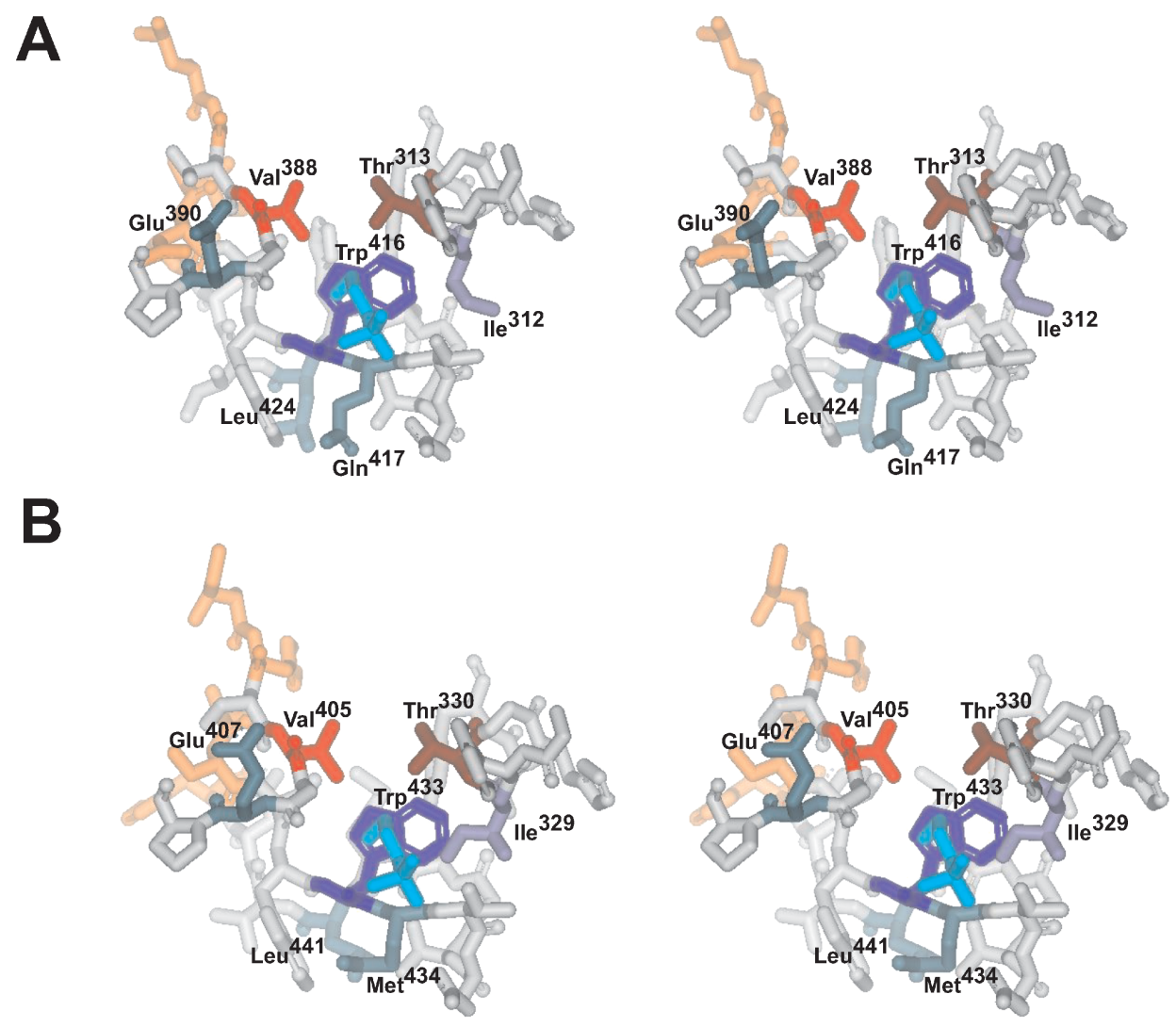

Figure 5. The acetate binding pocket of MT-ACS1 and AF-ACS2. (A) MT-ACS1 and (B) AF-ACS2 were modeled on the $S$. enterica ACS structure (PDB: 1PG4) using Accelrys DS Modeler 1.1 and the stereo image of the putative active site acetate binding pocket was created using DS ViewerPro 5.0. Residues within a $10 \AA$ A sphere of the propyl moiety of the propylphosphate group of the adenosine-5'-propylphosphate mimic of the acetyl-adenylate intermediate are shown. The acetate pocket residues $\left(\mathrm{Ile}^{312}, \mathrm{Thr}^{313}\right.$, $\mathrm{Val}^{388}$, and $\operatorname{Trp}^{416}$ of MT-ACS1 and $\mathrm{Ile}^{329}, \mathrm{Thr}^{330}, \mathrm{Val}^{405}$, and $\operatorname{Trp}^{433}$ of AF-ACS2) are colored and labeled. Those residues with high ConSurf scores that differ between MT-ACS1 and AF-ACS2 are shown in orange. Residues discussed in the text are labeled and shown in dark green. The propylphosphate group is shown in aqua.

hydrophobic pocket to better accommodate propionate and butyrate.

The results of our kinetic characterization of AF-ACS2 and the analysis of PA-ACS by Brasen et al. (2005), combined with phylogenetic analysis, sequence alignment, and structural modeling lead us to speculate that AF-ACS2 and PA-ACS, along with the Sulfolobus ACSs in group VIII in the phylogeny (Figure 4), represent a subclass of ACSs. We term these ACSs as "transitional," meaning that these enzymes have kinetic characteristics intermediate between traditional ACSs and propionyl-CoA synthetase and other short and medium chain acyl-CoA synthetases that show a preference for substrates other than acetate and have an expanded substrate range (Fujino et al. 2001a, Horswill and Escalante-Semerena 2002). The transitional ACSs would be expected to have only a slight preference for acetate over other substrates, but would also be expected to have a broader substrate range than traditional ACSs that utilize only acetate and propionate. Clearly, more evidence is necessary before the concept of transitional ACSs can be accepted. However, the accumulated data suggest a direction for further studies to prove or disprove this idea.

\section{Acknowledgments}

This work was supported by NIH (Award GM69374-01A1 to K.S. Smith), the South Carolina Experiment Station (Project SC-1700198 to K.S. Smith) and Clemson University. We thank Jean Pierre Touzel for providing genomic DNA from M. thermauto- trophicus Z245 and Imke Schroeder for providing genomic DNA from A. fulgidus. We also thank Kelcie Brunson for her technical assistance.

\section{References}

Allen, G.W.J. and S.H. Zinder. 1996. Methanogenesis from acetate by cell-free extracts of the thermophilic acetotrophic methanogen Methanothrix thermophila CALS-1. Arch. Microbiol. 166: 275-281.

Altschul, S.F., W. Gish, W. Miller, E.W. Myers and D.J. Lipman. 1990. Basic local alignment search tool. J. Mol. Biol. 215: 403-410.

Altschul, S.F., T.L. Madden, A.A. Schaffer, J. Zhang, Z. Zhang, W. Miller and D.J. Lipman. 1997. Gapped BLAST and PSIBLAST: a new generation of protein database search programs. Nucleic Acids Res. 25:3389-3402.

Anke, H. and L.B. Spector. 1975. Evidence for an acetyl-enzyme intermediate in the action of acetyl-CoA synthetase. Biochem. Biophys. Res. Commun. 67:767-773.

Armon, A., D. Graur and N. Ben-Tal. 2001. ConSurf: an algorithmic tool for the identification of functional regions in proteins by surface mapping of phylogenetic information. J. Mol. Biol. 307: 447-463.

Babbitt, P.C., G.L. Kenyon, B.M. Martin, H. Charest, M. Slyvestre, J.D. Scholten, K.H. Chang, P.H. Liang and D. Dunaway-Mariano. 1992. Ancestry of the 4-chlorobenzoate dehalogenase: analysis of amino acid sequence identities among families of acyl:adenyl ligases, enoyl-CoA hydratases/isomerases, and acyl-CoA thioesterases. Biochemistry 31:5594-5604. 
Berg, P. 1956a. Acyl adenylates: an enzymatic mechanism of acetate activation. J. Biol. Chem. 222:991-1013.

Berg, P. 1956b. Adenylates: the synthesis and properties of adenyl acetate. J. Biol. Chem. 222:1015-1034.

Bradford, M.M. 1976. A rapid and sensitive method for the quantitation of microgram quantities of protein utilizing the principle of protein-dye binding. Anal. Biochem. 72:248-254.

Brasen, C. and P. Schonheit. 2001. Mechanisms of acetate formation and acetate activation in halophilic archaea. Arch. Microbiol. 175:360-368.

Brasen, C. and P. Schonheit. 2004. Regulation of acetate and acetylCoA converting enzymes during growth on acetate and/or glucose in the halophilic archaeon Haloarcula marismortui. FEMS Microbiol. Lett. 241:21-26.

Brasen, C. and P. Schonheit. 2005. AMP-forming acetyl-CoA synthetase from the extremely halophilic archaeon Haloarcula marismortui: purification, identification and expression of the encoding gene, and phylogenetic affiliation. Extremophiles 9: 355-365.

Brasen, C., C. Urbanke and P. Schonheit. 2005. A novel octameric AMP-forming acetyl-CoA synthetase from the hyperthermophilic crenarchaeon Pyrobaculum aerophilum. FEBS Lett. 579:477-482.

Chang, K.H., H. Xiang and D. Dunaway-Mariano. 1997. Acyladenylate motif of the acyl-adenylate/thioester-forming enzyme superfamily: a site-directed mutagenesis study with the Pseudomonas sp. strain CBS3 4-chlorobenzoate:coenzyme A ligase. Biochemistry $36: 15,650-15,659$.

Conti, E., N.P. Franks and P. Brick. 1996. Crystal structure of firefly luciferase throws light on a superfamily of adenylate-forming enzymes. Structure 4:287-298.

Conti, E., T. Stachelhaus, M.A. Marahiel and P. Brick. 1997. Structural basis for the activation of phenylalanine in the non-ribosomal biosynthesis of gramicidin S. EMBO J. 16:4174-4183.

Eggen, R.I., A.C. Geerling, A.B. Boshoven and W.M. de Vos. 1991. Cloning, sequence analysis, and functional expression of the acetyl coenzyme A synthetase gene from Methanothrix soehngenii in Escherichia coli. J. Bacteriol. 173:6383-6389.

Fuchs, G., E. Stupperich and R.K. Thauer. 1978. Acetate assimilation and the synthesis of alanine, aspartate and glutamate in Methanobacterium thermoautotrophicum. Arch. Microbiol. 117: 61-66.

Fujino, T., J. Kondo, M. Ishikawa, K. Morikawa and T.T. Yamamoto. 2001a. Acetyl-CoA synthetase 2, a mitochondrial matrix enzyme involved in the oxidation of acetate. J. Biol. Chem. 276: $11,420-11,426$.

Fujino, T., Y.A. Takei, H. Sone, R.X. Ioka, A. Kamataki, K. Magoori, S. Takahashi, J. Sakai and T.T. Yamamoto. 2001b. Molecular identification and characterization of two medium-chain acyl-CoA synthetases, MACS1 and the Sa gene product. J. Biol. Chem. 276: 35,961-35,966.

Glaser, F., T. Pupko, I. Paz, R.E. Bell, D. Bechor-Shental, E. Martz and N. Ben-Tal. 2003. ConSurf: identification of functional regions in proteins by surface-mapping of phylogenetic information. Bioinformatics 19:163-164.

Grayson, N.A. and R.B. Westkaemper. 1988. Stable analogs of acyl adenylates. Inhibition of acetyl- and acyl-CoA synthetase by adenosine 5'-alkylphosphates. Life Sci. 43:437-444.

Gulick, A.M., V.J. Starai, A.R. Horswill, K.M. Homick and J.C. Escalante-Semerena. 2003. The $1.75 \AA$ crystal structure of acetyl-CoA synthetase bound to adenosine-5'-propylphosphate and coenzyme A. Biochemistry 42:2866-2873.

Horswill, A.R. and J.C. Escalante-Semerena. 2002. Characterization of the propionyl-CoA synthetase (PrpE) enzyme of Salmonella enterica: residue Lys592 is required for propionyl-AMP synthesis. Biochemistry 41:2379-2387.
Ingram-Smith, C., B.I. Woods and K.S. Smith. 2006. Characterization of the acyl substrate binding pocket of acetyl-CoA synthetase. Biochemistry. In press.

Jetten, M.S., A.J. Stams and A.J. Zehnder. 1989. Isolation and characterization of acetyl-coenzyme A synthetase from Methanothrix soehngenii. J. Bacteriol. 171:5430-5435.

Jogl, G. and L. Tong. 2004. Crystal structure of yeast acetylcoenzyme A synthetase in complex with AMP. Biochemistry 43: $1425-1431$.

Kleinkauf, H. and H. Von Dohren. 1996. A nonribosomal system of peptide biosynthesis. Eur. J. Biochem. 236:335-351.

Kumar, S., K. Tamura and M. Nei. 1994. MEGA: molecular evolutionary genetic analysis. Comput. Appl. Biosci. 10:189-191.

Laemmli, U.K. 1970. Cleavage of structural proteins during the assembly of the head of bacteriophage T4. Nature 227:680-685.

Landau, M., I. Mayrose, Y. Rosenberg, F. Glaser, E. Martz, T. Pupko and N. Ben-Tal. 2005. ConSurf 2005: the projection of evolutionary conservation scores of residues on protein structures. Nucleic Acids Res. 33:W299-302.

Lee, H.Y., K.B. Na, H.M. Koo and Y.S. Kim. 2001. Identification of active site residues in Bradyrhizobium japonicum acetyl-CoA synthetase. J. Biochem. (Tokyo) 130:807-813.

Lipmann, F. and L.C. Tuttle. 1945. A specific micromethod for determination of acyl phosphates. J. Biol. Chem. 159:21-28.

Luong, A., V.C. Hannah, M.S. Brown and J.L. Goldstein. 2000. Molecular characterization of human acetyl-CoA synthetase, an enzyme regulated by sterol regulatory element-binding proteins. J. Biol. Chem. 275:26,458-26,466.

Marahiel, M.A., T. Stachelhaus and H.D. Mootz. 1997. Modular peptide synthetases involved in nonribosomal peptide synthesis. Chem. Rev. 97:2651-2674.

May, J.J., N. Kessler, M.A. Marahiel and M.T. Stubbs. 2002. Crystal structure of DhbE, an archetype for aryl acid activating domains of modular nonribosomal peptide synthetases. Proc. Natl. Acad. Sci. USA 99:12,120-12,125.

Oberlies, G., G. Fuchs and R.K. Thauer. 1980. Acetate thiokinase and the assimilation of acetate in Methanobacterium thermoautotrophicum. Arch. Microbiol. 128:248-252.

Preston, G.G., J.D. Wall and D.W. Emerich. 1990. Purification and properties of acetyl-CoA synthetase from Bradyrhizobium japonicum bacteroids. Biochem. J. 267:179-183.

Rose, I.A., M. Grunberg-Manago, S.F. Korey and S. Ochoa. 1954. Enzymatic phosphorylation of acetate. J. Biol. Chem. 211: 737-756.

Stetter, K.O. 1988. Archaeoglobus fulgidus gen. nov., sp. nov.: a new taxon of extremely thermophilic archaebacteria. Syst. Appl. Microbiol. 10:172-173.

Teh, Y.L. and S.H. Zinder. 1992. Acetyl-coenzyme A synthetase in the thermophilic, acetate-utilizing methanogen Methanothrix sp. strain CALS-1. FEMS Microbiol. Lett. 98:1-8.

Thompson, J.D., T.J. Gibson, F. Plewniak, F. Jeanmougin and D.J. Higgins. 1997. The Clustal X windows interface: flexible strategies for multiple sequence alignment aided by quality analysis tools. Nucl. Acids Res. 25:4876-4882.

van den Berg, M.A., P. de Jong-Gubbels, C.J. Kortland, J.P. van Dijken, J.T. Pronk and H.Y. Steensma. 1996. The two acetylcoenzyme A synthetases of Saccharomyces cerevisiae differ with respect to kinetic properties and transcriptional regulation. J. Biol. Chem. 271:28,953-28,959.

Volkl, P., R. Huber, E. Drobner, R. Rachel, S. Burggraf, A. Trincone and K.O. Stetter. 1993. Pyrobaculum aerophilum sp. nov., a novel nitrate-reducing hyperthermophilic archaeum. Appl. Environ. Microbiol. 59:2918-2926. 
Webster, L.T., Jr. 1963. Studies of the acetyl coenzyme A synthetase reaction. I. Isolation and characterization of enzyme-bound acetyl adenylate. J. Biol. Chem. 238:4010-4015.
Zeikus, J.G. and R.S. Wolfe. 1972. Methanobacterium thermoautotrophicus sp. n., an anaerobic, autotrophic, extreme thermophile. J. Bacteriol. 109:707-715. 

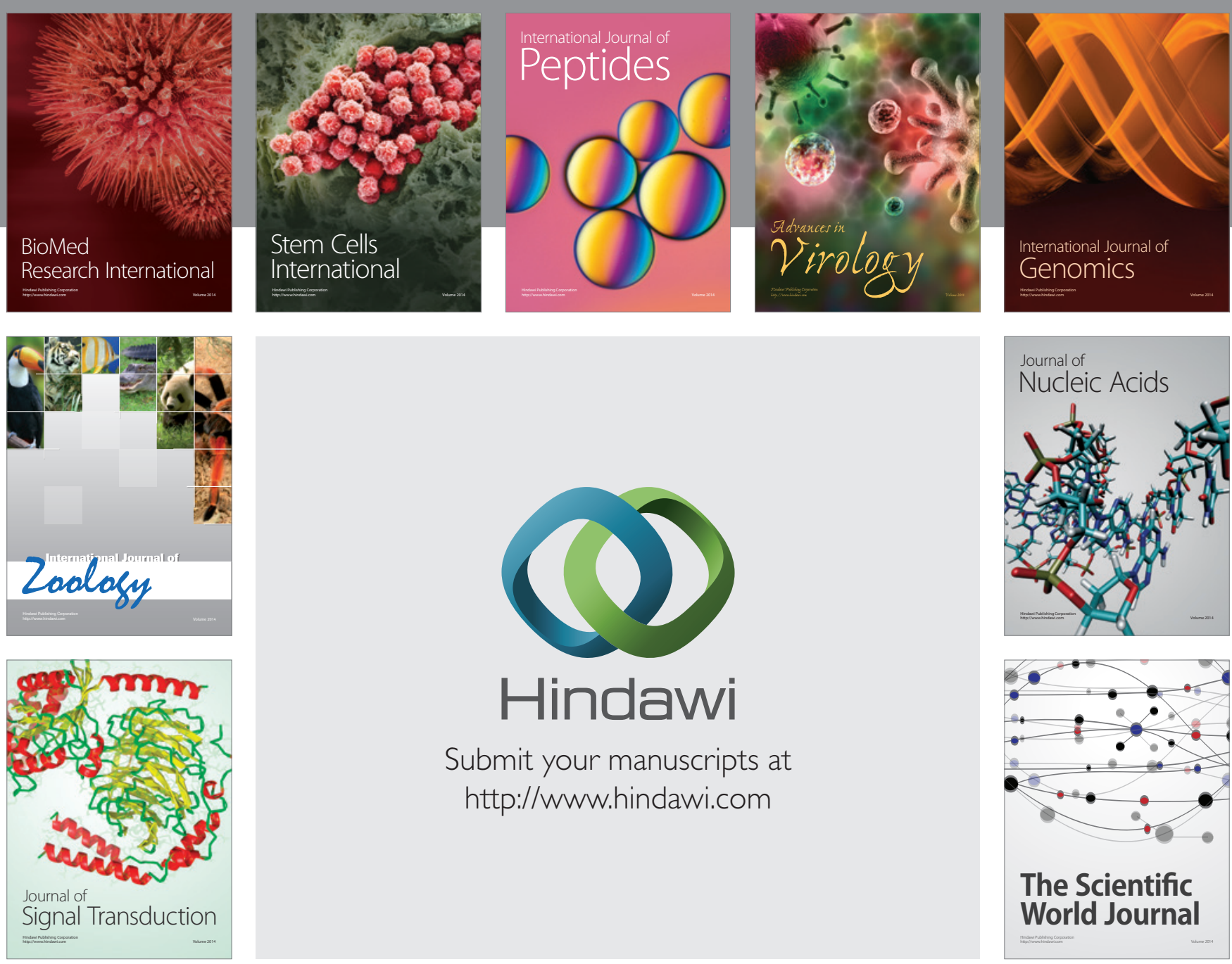

Submit your manuscripts at

http://www.hindawi.com
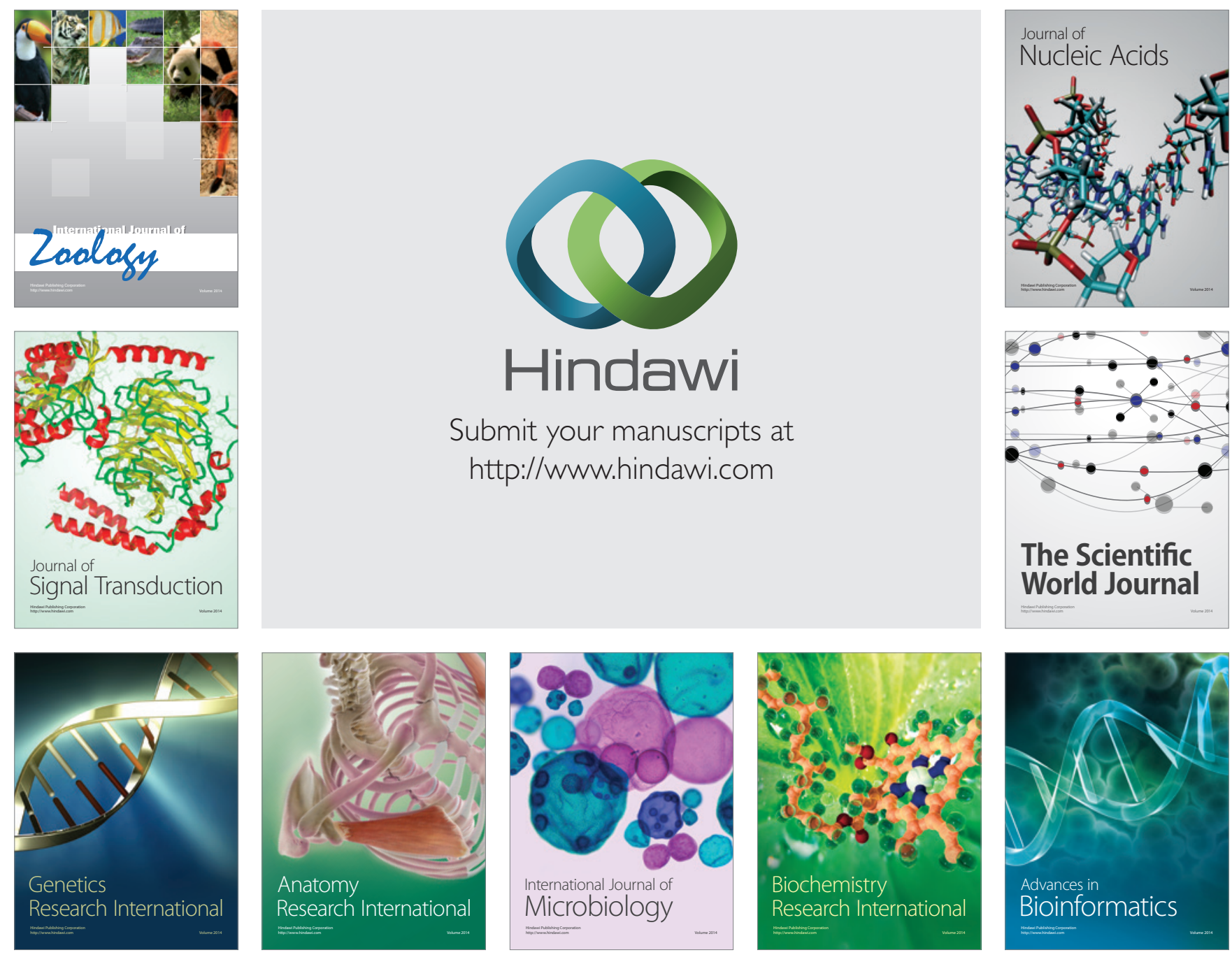

The Scientific World Journal
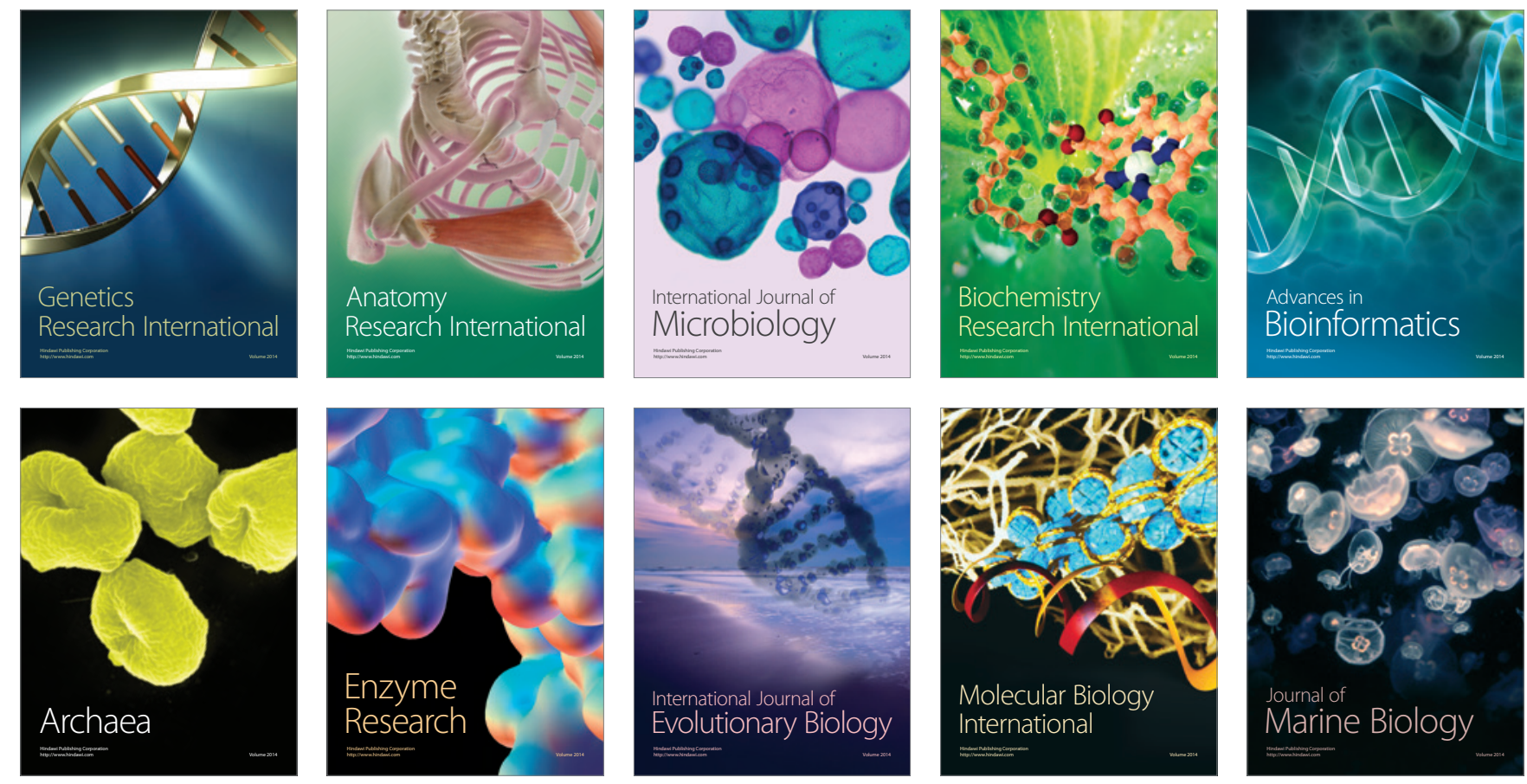